\title{
Transesophageal three-dimensional echocardiography improves the safety of transvenous extraction of pacemaker and implantable cardioverter-defibrillator leads - preliminary report
}

\author{
Maciej Kempa (D), Ludmila Daniłowicz-Szymanowicz (i), \\ Szymon Budrejko, Grzegorz Raczak
}

Department of Cardiology and Electrotherapy, Medical University of Gdańsk, Poland

\begin{abstract}
This article demonstrates the echocardiographic method to assess the placement of pacemaker and implantable cardioverter-defibrillator leads in the vicinity of the superior vena cava as a way to increase the safety of transvenous lead extraction (TLE). Three-dimensional transesophageal echocardiography was performed in 3 patients during TLE with the use of the Specranetics Excimer Laser device. We assessed the following parameters: lead adherence to the lateral wall of the vessel and lead movement. This method increased the safety of the performed TLE.

Keywords: transesophageal echocardiography • transvenous lead extraction • cardiac implantable electronic devices

\section{Citation}

Kempa M, Danilowicz-Szymanowicz L, Budrejko S, Raczak G. Transesophageal three-dimensional echocardiography improves the safety of transvenous extraction of pacemaker and implantable cardioverter-defibrillator leads - preliminary report. Eur J Transl Clin Med 2018;1(1):67-69. • DOI: 10.31373/ejtcm/94687
\end{abstract}

\section{Introduction}

An increasing number of patients with cardiovascular implantable electronic devices leads to more complications requiring transvenous lead extraction (TLE) as part of treatment [1]. Indications for this procedure are: damaged leads or a device-related infection (presenting as lead endocarditis or isolated infection of the device pocket) [2]. According to current guidelines, TLE procedures are considered to be complicated and should be performed only by experienced operators at reference centers with a cardiac surgery team on standby. Despite the advanced tools used in lead extraction, such as laser sheaths or mechanical sets with rotating cutters, intra- and immediate post-operative mortality from TLE still is noted0 [3-4]. Though relatively infrequent, the most dramatic complication is SVC or brachiocephalic vein damage, leading to massive bleeding into the mediastinum and often into the pleura. In such cases cardiosurgical intervention is the only life-saving treatment, though not always effective [4].

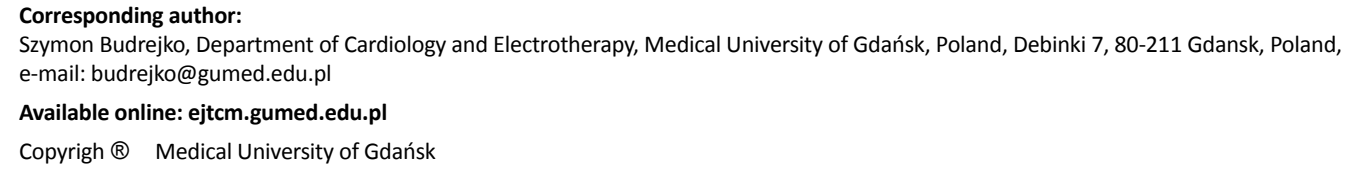

This is Open Access article distributed under the terms of the Creative Commons Attribution-ShareAlike 4.0 International (CC BY-SA 4.0); license available at: https:// creativecommons.org/licenses/by-sa/4.0/. 
A well-known risk factor of vessel damage during TLE is the type of lead undergoing extraction. Available data correlate greater risk with removing defibrillating leads, particularly those of dual-coil design [5-6]. The majority of complications are caused by coils placed in the SVC or its junction with the right atrium. While separating the lead from the vessel wall, an ingrown defibrillation coil might cause the sheath to damage the vessel. This risk appears to be particularly high when using laser sheaths and the lateral wall of the SVC is most prone to intraoperative injury. During transvenous extraction of leads that were implanted using access from the left side of the chest, the stiff sheath has a tendency to perforate the SVC in the direction towards the right lung. Therefore, any additional information about lead placement and the extent of its ingrowth into a particular wall of the vessel helps to identify patients with the greatest risk of vessel damage and resulting complications.

Transesophageal echocardiography (TEE) is a useful tool for confirming lead placement inside a vein, particularly when the device provides three-dimensional image reconstruction. Current guidelines describe various techniques of echocardiographic control of percutaneous procedures (e.g. transvenous closure of atrial septal defects, transcatheter aortic valve implantation, percutaneous mitral valve repair using MitraClip), however they do not discuss transvenous lead extraction [7-8].

\section{Methods}

We used a Spectrantics Excimer Laser System CVX300 and GE VIVID E95 ultrasound system (GE Ultrasound, Horten, Norway) equipped with a 6VT probe for echocardiographic assistance. Our cohort consisted of 2 women and $1 \mathrm{man}$, at the average age of 63 years (range 60-70 years of age). The indications for TLE were as follows: damaged defibrillation lead ( 2 cases) and infection of the device pocket (1 case). The leads were extracted after an average of 9 years since implantation (range: 7-11 years). We used GlideLight $16 \mathrm{~F}$ laser sheaths. All procedures were performed under general anesthesia with full cardiosurgical support available. No complications were noted.

After induction of anesthesia, TEE was performed intraoperatively. The initial 2D view was obtained at mid-esophageal level (probe at the depth of $25-40 \mathrm{~cm}$ ) at $80^{\circ}-110^{\circ}$ ("bicaval" view): superior vena cava - right atrium junction and the right atrium - inferior vena cava junction are visualized. Using the flexi-slice technique, a reconstructed image of the transverse section of SVC was obtained. Such 3D image made it possible to assess the lead placement (in relation to SVC walls) and its spontaneous movements during patient's cardiac and respiratory cycle (Figure 1).

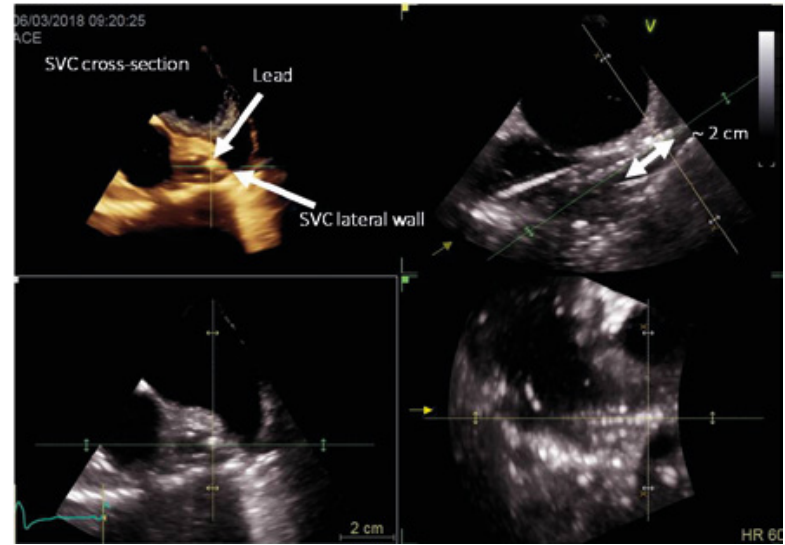

Figure 1. Example of 3D TOE zoom-mode aquisition of the transverse section at about $2 \mathrm{~cm}$ superior to the SVC-right atrium junction

After opening of the device pocket and dissecting the leads from adhering tissue, this technique allows the assessment of lead movement during the procedure. It is crucial to visualize the SVC at the most superior level possible, preferably including its initial segment where both brachiocephalic veins merge. If it is possible to see on the screen lead movement inside the SVC while the operator pulls on it, then it can be determined that it is safe to proceed with laser TLE (see Figure 2 A \& B).

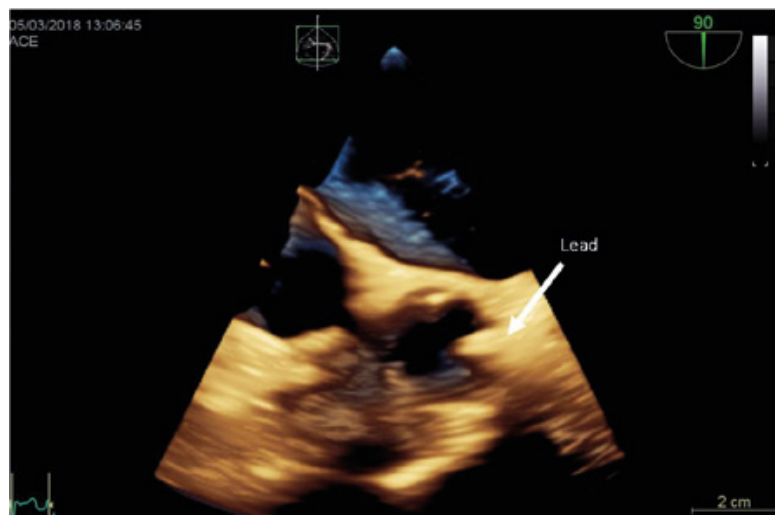

Figure 2. (A) Initial lead placement in the SVC

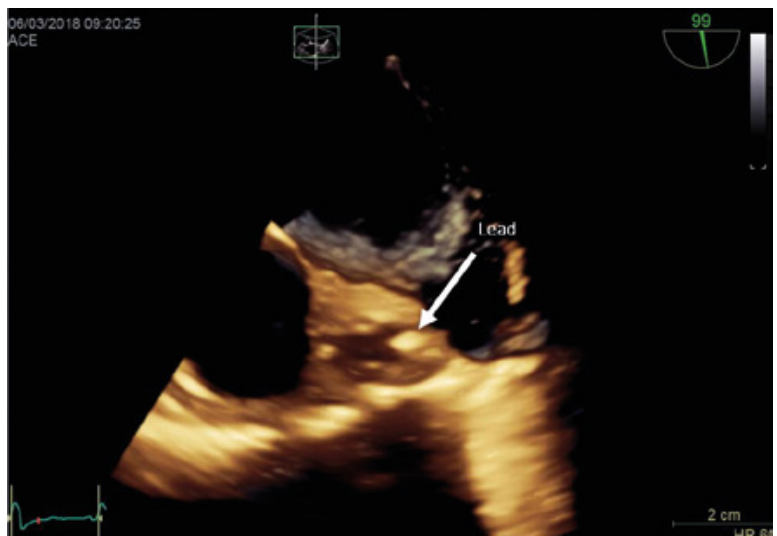

Figure 2. (B) Change of lead placement after pulling on it 
If pulling on the lead causes simultaneous movement of the SVC wall towards the midline, it indicates that the lead is ingrown into the vessel wall. In such cases, complications of TLE might be more likely, particularly if using laser sheaths. SVC wall damage may result in pericardial tamponade or extravasation of blood into the mediastinum or pleura. Intraoperative TEE allows the operator to assess the risk of SVC damage and to choose the best approach. Another advantage of oper- ating under the control of TEE is the ability to quickly diagnose complications, thus increasing the likelihood of a positive outcome.

Due to limited Publisher literature about the use of TEE to assess lead placement In the SVC during TLE, more studies are needed to confirm the efficacy of this method in reducing the rate of serious complications of TLE.

\section{References}

1. Polyzos KA, Konstantelias AA, Falagas ME. Risk factors for cardiac implantable electronic device infection: a systematic review and meta-analysis. Europace. 2015;17(5):767-777.

2. Kusumoto FM, Schoenfeld MH, Wilkoff BL, Berul Cl, Birgersdotter-Green UM, Carrillo R, et al. 2017 HRS expert consensus statement on cardiovascular implantable electronic device lead management and extraction. Hear Rhythm. 2017;14(12):e503-551.

3. Hauser RG, Katsiyiannis WT, Gornick CC, Almquist AK, Kallinen LM. Deaths and cardiovascular injuries due to device-assisted implantable cardioverter-defibrillator and pacemaker lead extraction. Europace. 2010;12(3):395-401.

4. Brunner MP, Cronin EM, Wazni O, Baranowski B, Saliba WI, Sabik JF, et al. Outcomes of patients requiring emergent surgical or endovascular intervention for catastrophic complications during transvenous lead extraction. Heart Rhythm. 2014 Mar;11(3):419-425.

5. Epstein LM, Love CJ, Wilkoff BL, Chung MK, Hackler JW, Bongiorni MG, et al. Superior vena cava defibrillator coils make transvenous lead extraction more challenging and riskier. J Am Coll Cardiol. 2013 Mar;61(9):987-989.

6. Brunner MP, Cronin EM, Duarte VE, Yu C, Tarakji KG, Martin DO, et al. Clinical predictors of adverse patient outcomes in an experience of more than 5000 chronic endovascular pacemaker and defibrillator lead extractions. Hear Rhythm. 2014;11(5):799-805.

7. Flachskampf FA, Wouters PF, Edvardsen T, Evangelista A, Habib G, Hoffman P, et al. Recommendations for transoesophageal echocardiography: EACVI update 2014. Eur Heart J Cardiovasc Imaging. 2014;15(4):353-365.

8. Hahn RT, Abraham T, Adams MS, Bruce CJ, Glas KE, Lang RM, et al. Guidelines for performing a comprehensive transesophageal echocardiographic examination: Recommendations from the american society of echocardiography and the society of cardiovascular anesthesiologists. Anesth Analg. 2014;118(1):21-68. 